\title{
TESTES DE VIGOR EM SEMENTES DE ALGODOEIRO HERBÁCEO ${ }^{1}$
}

\author{
ELISABETH APARECIDA FURTADO DE MENDONÇA², SUSIANE CORREA DE AZEVEDO³, \\ SEBASTIÃO CARNEIRO GUIMARÃES ${ }^{2}$, MARIA CRISTINA DE FIGUEIREDO E ALBUQUERQUE²
}

\begin{abstract}
RESUMO - O trabalho teve por objetivo avaliar a eficiência dos testes de deterioração controlada, de envelhecimento acelerado tradicional e modificado para avaliação do potencial fisiológico de sementes de algodoeiro, representadas por oito lotes da variedade CD 406. Para o teste de deterioração controlada, o grau de umidade das sementes foi ajustado para 15, 18, 21 e 24\%, e essas colocadas em banho-maria, por $24 \mathrm{~h}$, a 41, 43 e $45^{\circ} \mathrm{C}$. Para o teste de envelhecimento acelerado, foram utilizados os métodos tradicional, em câmaras com água, e modificado, com uso de solução saturada de $\mathrm{NaCl}$, ambos conduzidos sob três combinações de temperatura-tempo: $41^{\circ} \mathrm{C} / 48 \mathrm{~h}, 42^{\circ} \mathrm{C} / 60 \mathrm{~h}$ e $45^{\circ} \mathrm{C} / 72 \mathrm{~h}$. Conclui-se que os testes mais promissores para uso na avaliação do potencial fisiológico de sementes de algodoeiro são os testes de deterioração controlada com o teor de água das sementes ajustado artificialmente para $15 \%$, seguido de envelhecimento por $24 \mathrm{~h}$ em banho-maria a $43^{\circ} \mathrm{C}$ e o teste de envelhecimento acelerado com solução saturada de $\mathrm{NaCl}$, conduzido a $41^{\circ} \mathrm{C} / 48 \mathrm{~h}$. O envelhecimento acelerado tradicional a $45^{\circ} \mathrm{C} / 72 \mathrm{~h}$, mostrou-se muito drástico, provocando a perda da viabilidade das sementes.
\end{abstract}

Termos para indexação: Gossypium hirsutum L. r. latifolium Hutch, deterioração controlada, envelhecimento acelerado.

\section{VIGOR TESTS IN UPLAND COTTON SEEDS}

\begin{abstract}
The objective of this work was to study the efficiency of controlled deterioration and traditional and modified accelerated aging tests in order to evaluate the physiological potential of cotton seeds. Eight seed lots of cotton variety CD 406 were submitted to a controlled deterioration test, with seed moisture content adjusted to $15,18,21$, and $24 \%$. The seeds were submitted to aging in a double boiler at 41,43 , and $45^{\circ} \mathrm{C}$ for 24 hours. The accelerated aging test in chambers containing either water (traditional method) or saturated $\mathrm{NaCl}$ solution (modified method) was performed under three temperature-time conditions: $41^{\circ} \mathrm{C} / 48 \mathrm{~h}, 42^{\circ} \mathrm{C} / 60 \mathrm{~h}$, or $45^{\circ} \mathrm{C} / 72 \mathrm{~h}$. It was concluded that the controlled deterioration test, performed with seed moisture content artificially adjusted to $15 \%$, followed by aging in a double boiler at $43^{\circ} \mathrm{C}$ for 24 hours, as well as the accelerated aging test with the use of a saturated $\mathrm{NaCl}$ solution conducted at $41^{\circ} \mathrm{C} / 48 \mathrm{~h}$ are the most promising combinations to evaluate the physiological potential of cotton seeds. Traditional accelerated aging at $45^{\circ} \mathrm{C} / 72 \mathrm{~h}$ was exceedingly harsh on the seeds, causing seed viability loss
\end{abstract}

Index terms: Gossypium hirsutum L. r. latifolium Hutch, controlled deterioration, accelerated aging.

\footnotetext{
${ }^{1}$ Submetido em 18/10/2006. Aceito para publicação em 23/01/2007. Parte da dissertação de mestrado da segunda autora, apresentada a FAMEV/UFMT.
}

${ }^{2} \operatorname{Prof}(\mathrm{a})$. Dr(a) Departamento de Fitotecnia e Fitossanidade da FAMEV/UFMT, e-mail: beth@cpd.ufmt.br.

${ }^{3}$ Enga Agra, Mestre em Agricultura Tropical - Pesquisadora da COODETEC, MT.E-mail scazevedo@coodetec.com.br 


\section{INTRODUÇÃO}

Com a expansão e melhoria técnica da cultura do algodoeiro, a utilização de sementes de alta qualidade tornouse ainda mais relevante para o desempenho da lavoura, possibilitando a emergência uniforme no campo e a obtenção de plantas vigorosas, com reflexos diretos na produtividade.

$\mathrm{Na}$ determinação da qualidade de lotes de sementes, o teste de germinação é utilizado rotineiramente, mas por ser conduzido sob condições ambientais favoráveis, pode superestimar o potencial fisiológico dos mesmos. Por isso, foram desenvolvidos testes para estimar o vigor de lotes de sementes, procurando simular situações desfavoráveis às quais as sementes podem estar sujeitas (AOSA, 1983).

Os testes de vigor são capazes de detectar, com maior precisão, os avanços da deterioração das sementes, permitindo diferenciar lotes de poder germinativo semelhantes. Diversos estudos têm sido desenvolvidos para se testar o vigor, porém não existe método padronizado que possa ser recomendado para todas as espécies.

Além da necessidade de padronização de metodologias e de critérios para a interpretação de resultados, esses devem apresentar relação com a emergência de plântulas em campo, além de serem rápidos, objetivos, simples, de baixo custo e alta reprodutibilidade (AOSA, 1983). Dentre os testes já existentes, o de envelhecimento acelerado é um dos mais estudados para várias espécies cultivadas, sendo capaz de proporcionar informações com alto grau de consistência (TeKrony, 1995).

No Brasil, o envelhecimento acelerado é o principal teste empregado para avaliar o vigor de sementes de soja (Krzyzanowski e França Neto, 1991), e tem sido recomendado também para outras espécies como feijão (Dias e Barros, 1992), milho (Barros e Dias, 1992), trigo (Irigon e Rossini, 1992) e algodoeiro (Laposta, 1991; Torres, 1998).

No entanto, um único teste de vigor não é capaz de caracterizar todas as interações possíveis entre as sementes e as condições ambientais predominantes antes, durante e após a colheita, bem com as verificadas por ocasião da semeadura (Hampton e Coolbear, 1990). Nesse sentido, outros testes de vigor precisam ser utilizados para aumentar as informações e diminuir os erros associados à decisão de se aceitar ou rejeitar um lote de sementes para armazenamento ou semeadura.

Uma das deficiências do teste de envelhecimento acelerado é a diferença na velocidade de absorção de água entre sementes grandes e pequenas (Panobianco e Marcos Filho, 2001), causando desuniformidade na amostra, o que pode ser minimizado utilizando-se soluções salinas saturadas em substituição à água, dentro do ambiente de envelhecimento (Jianhua e McDonald, 1996). Esse fato foi comprovado em estudos com sementes de Impatiens wallerana (Jianhua e McDonald, 1996), e essa técnica também apresentou resultados mais consistentes em outras espécies como pimentão (Panobianco e Marcos Filho, 1998), milho doce (Bennett et al., 1998), cenoura (Rodo et al., 2000) e brócoli (Mendonça et al., 2000). Nesse teste, ajustase o grau de umidade das sementes para, no mínimo, 15,5\%, antes de submetê-las às altas temperaturas, que geralmente variam entre 40 e $45^{\circ} \mathrm{C}$ (Torres, 2002). Como a capacidade de absorção de água pelas sementes varia com a espécie e diferentes tipos de sais condicionam umidades relativas distintas, o teste precisa ser adaptado para cada espécie.

Apesar dos resultados consistentes para a avaliação do vigor de lotes de sementes de olerícolas e forrageiras, o teste de deterioração controlada tem sido pouco estudado em grandes culturas, não obstante o amplo potencial de uso (Rossetto e Marcos Filho, 1995).

No caso específico do algodoeiro, são poucos os estudos sobre testes de vigor para avaliação do potencial fisiológico de suas sementes. Assim, objetivou-se com esta pesquisa adequar os testes de deterioração controlada e de envelhecimento acelerado, tradicional e modificado, para a avaliação do vigor de sementes de algodoeiro herbáceo.

\section{MATERIAL E MÉTODOS}

O trabalho foi conduzido no Laboratório de Análise de Sementes da Faculdade de Agronomia e Medicina Veterinária da Universidade Federal de Mato Grosso, utilizando-se oito lotes comerciais de sementes de algodoeiro, variedade CD 406, produzidas no município de Primavera do Leste, MT, pela Cooperativa Central de Pesquisa Agrícola (COODETEC). As sementes de cada lote foram homogeneizadas e mantidas em embalagens de papel "Kraft", em câmara climatizada (cerca de $18^{\circ} \mathrm{C}$ e $65 \%$ UR), até a realização das análises.

O grau de umidade foi determinado, antes e após o período de deterioração controlada e do envelhecimento acelerado, pelo método da estufa a $105 \pm 3^{\circ} \mathrm{C}$, durante 24h, de acordo com as Regras para Análise de Sementes (Brasil, 1992), utilizando-se duas subamostras com aproximadamente 5,0g de sementes. Os resultados foram expressos em porcentagem média (base úmida). Os testes de germinação e primeira contagem foram conduzidos com 400 sementes, distribuídas em oito subamostras de 50 sementes, colocadas para germinar em rolo de papel germitest, umedecido com água destilada na quantidade de 
2,5 vezes a sua massa. Os rolos foram mantidos em câmara de germinação, a $25^{\circ} \mathrm{C}$, com $8 \mathrm{~h}$ de luz por dia. A primeira contagem de germinação constituiu-se da porcentagem de plântulas normais obtidas aos quatro dias após a semeadura, e a germinação, da porcentagem de plântulas normais aos 12 dias (Brasil, 1992). A germinação em baixa temperatura foi determinada seguindo-se o procedimento descrito para o teste de germinação, sendo os rolos de papel mantidos em incubadora B.O.D a $18^{\circ} \mathrm{C} \pm 0,5^{\circ} \mathrm{C}$, permanecendo no escuro até a contagem única realizada aos sete dias (AOSA, 1983; ISTA, 1995). As plântulas consideradas normais foram as que apresentaram comprimento $\geq$ a $4 \mathrm{~cm}$. A emergência de plântulas em campo foi conduzida com 400 sementes, distribuídas em oito subamostras de 50 sementes para cada lote, semeadas a $5 \mathrm{~cm}$ de profundidade, em linhas de $2,0 \mathrm{~m}$ de comprimento, distanciadas de $0,20 \mathrm{~m}$ entre si, em Latossolo Vermelho Amarelo, textura média, cultivado anteriormente com algodoeiro. O teste foi conduzido em dezembro de 2003, no município de Primavera do Leste, MT, situado a $15^{\circ} 33^{\prime} 45^{\prime \prime}$ latitude sul e $54^{\circ} 17^{\prime} 41,8^{\prime \prime}$ longitude oeste, com altitude de $636 \mathrm{~m}$, precipitação média de $2000 \mathrm{~mm}$.ano ${ }^{-1}$ e temperatura média anual de $24^{\circ} \mathrm{C}$. As avaliações foram efetuadas de acordo com Nakagawa (1999), computando-se o número de plântulas normais emersas aos 14 dias após a semeadura. Para o teste de deterioração controlada foram pesadas aproximadamente $28 \mathrm{~g}$ de sementes de cada lote, cujo grau de umidade, base úmida, foi elevado artificialmente para quatro níveis distintos: $15 \%, 18 \%, 21 \%$ e $24 \%$, pelo método do rolo de papel, umedecido previamente com água destilada na proporção de 2,25:1, de forma que as sementes ficassem distribuídas sobre duas folhas e cobertas com a terceira. Os rolos foramcolocadosemcaixas plásticascomtampa,mantidos em câmara de germinação a $20^{\circ} \mathrm{C}$ e monitorados mediante pesagens sucessivas das sementes até a obtenção dos valores de umidade desejados (Hampton e TeKrony, 1995b). Para facilitar os trabalhos de laboratório, antecedendo essa etapa foi determinada a curva de absorção de água pelas sementes, por meio da qual foram obtidos os tempos prováveis para a obtenção dos teores de água requeridos, que foram de 3, 4, 5 , e $7 \mathrm{~h}$. Uma vez obtidos os graus de umidade planejados, cada amostra foi colocada em embalagem laminada de alumínio + plástico, fechada hermeticamente em seladora elétrica, acondicionadas em dessecador e mantidas por $24 \mathrm{~h}$ em câmara refrigerada $\left( \pm 8^{\circ} \mathrm{C}\right.$ e $\left.65 \% \mathrm{UR}\right)$, com a finalidade de assegurar a distribuição uniforme de água no interior das sementes. Em seguida, as amostras foram mantidas por $24 \mathrm{~h}$ em banho-maria calibrado para as temperaturas de $41^{\circ} \mathrm{C}, 43^{\circ} \mathrm{C}$ e $45^{\circ} \mathrm{C}$. Ao término desse período, ficaram em dessecador por 30 minutos para reduzir a temperatura, foram retiradas da embalagem, pesadas para a determinação do grau de umidade e imediatamente submetidas ao teste de germinação (Powell, 1995). A avaliação foi efetuada quatro dias após a semeadura, computando-se a porcentagem média de plântulas normais. Para o teste de envelhecimento acelerado foi empregado o método tradicional (com água) e o alternativo (com solução salina saturada de cloreto de sódio - $\mathrm{NaCl}$ ). O teste foi conduzido utilizando-se caixa de plástico (gerbox) possuindo em seu interior suporte de tela metálica para apoio, sobre a qual foram distribuídas cerca de $28 \mathrm{~g}$ de sementes de cada lote. Dentro das caixas foram colocados $40 \mathrm{~mL}$ de água destilada ou, no teste modificado, solução salina saturada de $\mathrm{NaCl}$. As caixas tampadas foram mantidas em incubadoras tipo B.O.D reguladas a $41^{\circ} \mathrm{C} / 48 \mathrm{~h}$ (Marcos Filho, 1999b); $42^{\circ} \mathrm{C} / 60 \mathrm{~h}$ (Laposta, 1991) e $45^{\circ} \mathrm{C} / 72 \mathrm{~h}$ (ISTA, 1995). Decorrido cada período, quatro subamostras de 50 sementes por tratamento foram colocadas para germinar, seguindo o mesmo procedimento utilizado para o teste de germinação. A avaliação foi realizada aos quatro dias após a semeadura, computando-se a porcentagem de plântulas com comprimento $\geq 4 \mathrm{~cm}$. A solução saturada de $\mathrm{NaCl}$ foi preparada tendo como solvente a água destilada, nas temperaturas de $41^{\circ} \mathrm{C}, 42^{\circ} \mathrm{C}$ e $45^{\circ} \mathrm{C}$, correspondentes àquelas de realização dos testes de envelhecimento. O sal utilizado foi o cloreto de sódio cristalizado para análise $(99,5 \%$ de $\mathrm{NaCl}$,). Nessas condições espera-se umidade relativa do ar no interior das câmaras de 76\% (Jianhua e McDonald, 1996).

Todos os testes foram realizados segundo o delineamento inteiramente casualizado, com oito repetições para os testes de germinação, primeira contagem da germinação, germinação a baixa temperatura e emergência em campo; e quatro repetições para os testes de deterioração controlada e envelhecimento acelerado. Os dados foram submetidos à análise de variância, e as médias comparadas pelo teste de Scott e Knott a $10 \%$ de probabilidade.

\section{RESULTADOS E DISCUSSÃO}

Na Tabela 1 são observados os resultados da caracterização inicial dos oito lotes de sementes de algodoeiro. Os lotes avaliados apresentaram teor de água entre $8,9 \mathrm{e}$ $9,3 \%$, e essa variação máxima de 0,4 pontos percentuais são indicativas da uniformidade entre lotes, necessária para que os resultados expressem o efeito dos tratamentos. De acordo com Marcos Filho (2005), deve haver controle rigoroso na execução do teste de grau de umidade, e a variação máxima no teor de água entre os lotes deve ser de $2 \%$. 
TABELA 1. Valores médios do grau de umidade (GU), teste padrão de germinação (TPG), primeira contagem da germinação (PCG), teste de germinação a baixa temperatura (GBT) e emergência em campo (EC) de oito lotes de sementes de algodoeiro.

\begin{tabular}{|c|c|c|c|c|c|}
\hline \multirow{2}{*}{ Lote } & GU & TG & PCG & GBT & EC \\
\hline & \multicolumn{5}{|c|}{ 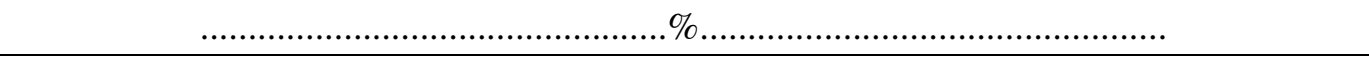 } \\
\hline 1 & 8,9 & $74 \mathrm{~A}$ & $70 \mathrm{~B}$ & $63 \mathrm{~A}$ & $54 \mathrm{~A}$ \\
\hline 2 & 9,1 & $72 \mathrm{~A}$ & $70 \mathrm{~B}$ & $51 \mathrm{~B}$ & $67 \mathrm{~A}$ \\
\hline 3 & 9,2 & $81 \mathrm{~A}$ & $79 \mathrm{~A}$ & $58 \mathrm{~A}$ & $49 \mathrm{~B}$ \\
\hline 4 & 8,9 & $82 \mathrm{~A}$ & $82 \mathrm{~A}$ & $64 \mathrm{~A}$ & $58 \mathrm{~A}$ \\
\hline 5 & 8,9 & $76 \mathrm{~A}$ & $66 \mathrm{~B}$ & $63 \mathrm{~A}$ & $59 \mathrm{~A}$ \\
\hline 6 & 8,9 & $78 \mathrm{~A}$ & $74 \mathrm{~A}$ & $65 \mathrm{~A}$ & $57 \mathrm{~A}$ \\
\hline 7 & 8,9 & $79 \mathrm{~A}$ & $77 \mathrm{~A}$ & $50 \mathrm{~B}$ & $49 \mathrm{~B}$ \\
\hline 8 & 9,3 & $77 \mathrm{~A}$ & $77 \mathrm{~A}$ & $43 \mathrm{~B}$ & $42 \mathrm{~B}$ \\
\hline
\end{tabular}

Médias nas colunas, seguidas pela mesma letra, não diferem entre si pelo teste de Scott e Knott em 10\% de probabilidade.

Os lotes tiveram porcentagem de germinação semelhante, com valores entre 72 e $82 \%$, e ficaram dentro do padrão para a comercialização para o estado de Mato Grosso (CESM, 2005), cujo limite mínimo é 70\%. Resultados semelhantes de germinação é também requisito necessário para inclusão dos lotes nas avaliações dos testes de vigor. Lotes com mesma germinação não necessariamente possuem mesmo vigor, uma vez que o teste de germinação é conduzido em condições favoráveis, permitindo a expressão do potencial máximo para produzir plântulas normais (Marcos Filho, 1999a). Hampton e TeKrony (1995a) também afirmaram que a maior limitação do teste de germinação é sua inabilidade para detectar diferenças de vigor entre lotes com alta germinação, indicando a necessidade de complementar essa informação. Desse modo, os lotes foram considerados adequados para serem comparados quanto ao vigor, uma vez que tiveram grau de umidade e porcentagens de germinação semelhantes.

Os valores obtidos na primeira contagem do teste de germinação foram, para a maioria dos lotes, iguais ou semelhantes à germinação, indicando que a totalidade, ou a grande parte das sementes germinadas, já tinham formado plântulas normais quatro dias após a semeadura. Com base nesse teste, o lote 5 foi o de menor vigor, seguido pelos lotes 1 e 2 .

Por outro lado, nos testes de germinação a baixa temperatura e emergência em campo os valores obtidos foram inferiores à germinação, e, nos dois casos, os lotes 7 e 8 ficaram classificados como de menor vigor. Assim, configurou-se a dificuldade conhecida na separação de lotes com intermediário vigor, pois os mesmos estão sujeitos a variações em função da metodologia empregada, principalmente quando se trata de lotes com diferenças pouco acentuadas. Por isso é necessária a utilização de mais de um teste de vigor para a determinação do potencial fisiológico desses lotes (Marcos Filho, 2005).

O teor de água das sementes, antes e após a deterioração controlada (Tabela 2), variou de 0,0 a 0,2 pontos porcentuais entre lotes, dentro de cada nível de umidade, situando-se dentro de limites toleráveis. Individualmente, a variação entre os valores médios de umidade ajustados para 15,18 , 21 e $24 \%$, entre lotes foram de 0,$2 ; 0,2 ; 0,2$ e 0,1 pontos porcentuais, respectivamente. Esses resultados corroboram as afirmações que, no teste de deterioração controlada, o teor de água das sementes é mantido durante o envelhecimento (Rosseto e Marcos Filho, 1995; Krzyzanowski e Vieira, 1999).

O comportamento dos lotes, quanto à germinação após o teste de deterioração controlada, foi dependente da interação entre o teor de água inicial das sementes e a temperatura de realização do teste (Tabela 3). Dentro de cada lote, o grau de deterioração, expresso pela porcentagem de germinação, foi tanto maior quanto maiores foram o teor de água inicial das sementes e a temperatura de execução do teste. Nas combinações de alta umidade com alta temperatura $\left(24 \% / 45^{\circ} \mathrm{C}, 24 \% / 43^{\circ} \mathrm{C}\right.$ e $\left.21 \% / 45^{\circ} \mathrm{C}\right)$, o efeito sobre as sementes foi drástico, ocorrendo reduções na germinação de mais de 50 pontos percentuais em relação às sementes não deterioradas, situação em que a diferença entre lotes de diferentes níveis de vigor pode deixar de ser evidenciada. 
Esses resultados concordam com as afirmações de Marcos Filho (2005), de que sementes mais úmidas mostram maior sensibilidade às condições de alta temperatura, pois apresentam atividade metabólica intensificada sob condições de envelhecimento. Esse fato já foi confirmado também no teste de deterioração controlada (Rossetto e Marcos Filho, 1995).

TABELA 2. Grau de umidade de oito lotes de sementes ajustados artificialmente para 15, 18, 21 e $24 \%$, para execução do teste de deterioração controlada em sementes de algodoeiro.

\begin{tabular}{|c|c|c|c|c|c|}
\hline \multirow{3}{*}{ Lote } & \multirow{2}{*}{ GUi } & \multicolumn{4}{|c|}{ Grau de umidade ajustado } \\
\hline & & 15 & 18 & 21 & 24 \\
\hline & \multicolumn{5}{|c|}{ 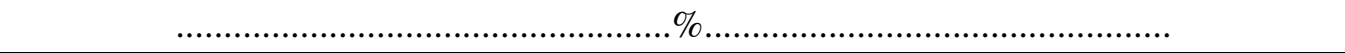 } \\
\hline 1 & 10,0 & 15,0 & 18,2 & 21,2 & 24,0 \\
\hline 2 & 9,9 & 15,0 & 18,0 & 21,0 & 24,0 \\
\hline 3 & 10,1 & 15,2 & 18,0 & 21,0 & 24,0 \\
\hline 4 & 10,2 & 15,2 & 18,0 & 21,0 & 24,0 \\
\hline 5 & 9,9 & 15,1 & 18,2 & 21,1 & 24,0 \\
\hline 6 & 10,1 & 15,0 & 18,0 & 21,0 & 24,1 \\
\hline 7 & 10,1 & 15,0 & 18,0 & 21,0 & 24,1 \\
\hline 8 & 10,5 & 15,1 & 18,0 & 21,2 & 24,1 \\
\hline Média & 10,1 & 15,1 & 18,1 & 21,1 & 24,0 \\
\hline
\end{tabular}

$\mathrm{GUi}=$ grau de umidade inicial das sementes antes do ajuste artificial.

TABELA 3. Porcentagem de germinação de oito lotes de sementes de algodoeiro, com teores iniciais de água de $15 \%$, $18 \%, 21 \%$ e $24 \%$, após 24 h de deterioração controlada (DC) nas temperaturas de 41,43 e $45^{\circ} \mathrm{C}$.

\begin{tabular}{|c|c|c|c|c|c|c|c|c|c|c|c|c|}
\hline \multirow[b]{2}{*}{ Lote } & \multicolumn{3}{|c|}{$15 \%$} & \multicolumn{3}{|c|}{$18 \%$} & \multicolumn{3}{|c|}{$21 \%$} & \multicolumn{3}{|c|}{$24 \%$} \\
\hline & $41^{\circ} \mathrm{C}$ & $43^{\circ} \mathrm{C}$ & $45^{\circ} \mathrm{C}$ & $41^{\circ} \mathrm{C}$ & $43^{\circ} \mathrm{C}$ & $45^{\circ} \mathrm{C}$ & $41^{\circ} \mathrm{C}$ & $43^{\circ} \mathrm{C}$ & $45^{\circ} \mathrm{C}$ & $41^{\circ} \mathrm{C}$ & $43^{\circ} \mathrm{C}$ & $45^{\circ} \mathrm{C}$ \\
\hline 1 & $74 \mathrm{~A}$ & $63 \mathrm{~A}$ & $60 \mathrm{~A}$ & $71 \mathrm{~A}$ & 60B & $59 \mathrm{~A}$ & $65 \mathrm{C}$ & $59 \mathrm{~A}$ & $48 \mathrm{~A}$ & $71 \mathrm{~A}$ & $41 \mathrm{~B}$ & $36 \mathrm{~A}$ \\
\hline 2 & $64 \mathrm{~B}$ & $58 \mathrm{~B}$ & $55 \mathrm{~A}$ & $67 \mathrm{~B}$ & $60 \mathrm{~B}$ & $55 \mathrm{~A}$ & $58 \mathrm{D}$ & 47B & $42 B$ & $66 \mathrm{~B}$ & $41 \mathrm{~B}$ & $25 \mathrm{~B}$ \\
\hline 3 & $73 \mathrm{~A}$ & $66 \mathrm{~A}$ & $55 \mathrm{~A}$ & $75 \mathrm{~A}$ & $66 \mathrm{~A}$ & $60 \mathrm{~A}$ & $82 \mathrm{~A}$ & $61 \mathrm{~A}$ & $44 B$ & $74 \mathrm{~A}$ & $51 \mathrm{~A}$ & $35 \mathrm{~A}$ \\
\hline 4 & $76 \mathrm{~A}$ & $72 \mathrm{~A}$ & $60 \mathrm{~A}$ & 67B & $64 \mathrm{~A}$ & $57 \mathrm{~A}$ & $66 \mathrm{C}$ & $58 \mathrm{~A}$ & $52 \mathrm{~A}$ & $53 \mathrm{C}$ & $54 \mathrm{~A}$ & $38 \mathrm{~A}$ \\
\hline 5 & $77 \mathrm{~A}$ & $68 \mathrm{~A}$ & $59 \mathrm{~A}$ & $71 \mathrm{~A}$ & $57 \mathrm{~B}$ & $52 \mathrm{~A}$ & $72 B$ & $45 \mathrm{~B}$ & $47 \mathrm{~A}$ & $67 \mathrm{~B}$ & $41 B$ & $40 \mathrm{~A}$ \\
\hline 6 & $74 \mathrm{~A}$ & $68 \mathrm{~A}$ & $56 \mathrm{~A}$ & $75 \mathrm{~A}$ & $64 \mathrm{~A}$ & $52 \mathrm{~A}$ & $57 \mathrm{D}$ & $51 \mathrm{~B}$ & $40 \mathrm{~B}$ & $66 \mathrm{~B}$ & $36 \mathrm{C}$ & $37 \mathrm{~A}$ \\
\hline 7 & $75 \mathrm{~A}$ & $56 \mathrm{~B}$ & $55 \mathrm{~A}$ & $69 B$ & $55 \mathrm{~B}$ & $54 \mathrm{~A}$ & $64 \mathrm{C}$ & 49B & $46 \mathrm{~A}$ & $57 \mathrm{C}$ & $46 \mathrm{~A}$ & $42 \mathrm{~A}$ \\
\hline 8 & $64 \mathrm{~B}$ & $44 \mathrm{C}$ & $42 \mathrm{~B}$ & $65 \mathrm{~B}$ & $37 \mathrm{C}$ & $35 \mathrm{~B}$ & $57 \mathrm{D}$ & $37 \mathrm{C}$ & $30 \mathrm{C}$ & $54 \mathrm{C}$ & $31 \mathrm{C}$ & $29 B$ \\
\hline
\end{tabular}

Em cada coluna, médias seguidas pela mesma letra não diferem entre si pelo teste de Scott e Knott em 10\% de probabilidade.

Na temperatura de $43^{\circ} \mathrm{C}$, nos teores de água de $15 \%$, $18 \%$ e $21 \%$, os lotes foram separados em três classes, estando o lote 8 classificado sempre como o de pior vigor, os lotes 2 e 7 como de vigor intermediário e os lotes 1,3 e 4 como de maior vigor (Tabela 3 ). Nas outras combinações de umidade e temperatura, o lote 8 foi confirmado como sendo o de menor vigor, os lotes 2 e 7 como de menor vigor ou vigor intermediário e o lote 3 como o de melhor vigor. À exceção da combinação $21 \% / 41^{\circ} \mathrm{C}$, em que o lote 3 foi classificado isoladamente como o de melhor vigor, os lotes 1, 4 ou 1 e 4 também foram ratificados como de melhor vigor. Em relação aos testes de vigor realizados inicialmente (Tabela 1), a deterioração controlada permitiu maior estratificação dos lotes em níveis de vigor, tendo classificado os lotes de pior e melhor vigor de maneira semelhante ao teste de germinação a baixa temperatura, e também apresentando alguma similaridade ao teste de emergência a campo. Há que se ressaltar, no entanto, a discrepância observada na separação 
do lote 3, classificado como de menor vigor na emergência a campo, e como de melhor vigor em todas as situações na deterioração controlada. O tipo e intensidade do estresse aos quais as sementes ficam submetidas a campo são variáveis, podendo os lotes ser classificados de maneira diferente em testes distintos. Esse fato justifica a necessidade de mais de um teste de vigor no processo de julgamento de lotes de sementes para comercialização ou armazenamento. Dada a grande estabilidade nos resultados, e a separação dos lotes em classes similares a testes já muito utilizados, a deterioração controlada apresenta potencial para uso na classificação de lotes de sementes de algodoeiro, sendo recomendados estudos adicionais com outros lotes e variedades.

É oportuno ressaltar que, o teste de deterioração controlada, embora inicialmente recomendado para sementes de hortaliças (Powell e Matthews, 1981), tem sido adaptado por pesquisadores brasileiros (Rossetto e Marcos Filho, 1995; Marcos Filho et al., 2001; Santos et al., 2003; Rosseto et al., 2004) para sementes de grandes culturas como soja, feijão e amendoim, com resultados promissores, a exemplo do presente trabalho com algodoeiro.

Os dados referentes ao grau de umidade das sementes, após a realização do teste de envelhecimento acelerado com procedimento tradicional e com solução saturada de $\mathrm{NaCl}$, são apresentados na Tabela 4. A variação no grau de umidade das sementes entre lotes, numa mesma condição de envelhecimento, foi de, no máximo, 1,7 pontos percentuais, dentro dos limites considerados aceitáveis para a validade dos resultados do teste, que é de 2\% (Marcos Filho, 2005).

TABELA 4. Grau de umidade (\%) de oito lotes de sementes após envelhecimento acelerado (EA) com $\mathrm{H}_{2} \mathrm{O}$ e solução saturada de $\mathrm{NaCl}$, em três combinações de temperatura e períodos de exposição.

\begin{tabular}{|c|c|c|c|c|c|c|}
\hline & \multicolumn{3}{|c|}{$\mathrm{EA}-\mathrm{H}_{2} \mathrm{O}$} & \multicolumn{3}{|c|}{$\mathrm{EA}-\mathrm{NaCl}$} \\
\hline & $41^{\circ} \mathrm{C} / 48 \mathrm{~h}$ & $42^{\circ} \mathrm{C} / 60 \mathrm{~h}$ & $45^{\circ} \mathrm{C} / 72 \mathrm{~h}$ & $41^{\circ} \mathrm{C} / 48 \mathrm{~h}$ & $42^{\circ} \mathrm{C} / 60 \mathrm{~h}$ & $45^{\circ} \mathrm{C} / 72 \mathrm{~h}$ \\
\hline 1 & 22,2 & 25,2 & 26,3 & 11,0 & 11,8 & 12,0 \\
\hline 2 & 21,8 & 25,5 & 26,7 & 11,0 & 11,8 & 12,0 \\
\hline 3 & 22,6 & 24,7 & 26,3 & 11,0 & 11,5 & 12,0 \\
\hline 4 & 22,8 & 24,7 & 26,0 & 11,0 & 11,5 & 12,0 \\
\hline 5 & 22,7 & 24,1 & 26,6 & 11,0 & 11,4 & 12,0 \\
\hline 6 & 23,0 & 24,5 & 25,0 & 10,5 & 12,0 & 12,0 \\
\hline 7 & 22,9 & 23,8 & 26,7 & 10,4 & 11,7 & 12,0 \\
\hline 8 & 23,0 & 24,1 & 25,5 & 11,1 & 11,7 & 12,4 \\
\hline Média & 22,6 & 24,6 & 26,1 & 10,9 & 11,7 & 12,1 \\
\hline Amplitude & 1.2 & 1.7 & 1.7 & 0.7 & 0.6 & 0.4 \\
\hline
\end{tabular}

No envelhecimento com solução saturada de $\mathrm{NaCl}$, a absorção de água pelas sementes de algodoeiro foi muito baixa, variando o teor de água, na média dos lotes, de $10,9 \%$ para a condição $41^{\circ} \mathrm{C} / 48 \mathrm{~h}$ a $12,4 \%$ na condição $45^{\circ} \mathrm{C} / 72 \mathrm{~h}$, apresentando também menor variação de umidade entre lotes. O uso de solução saturada de $\mathrm{NaCl}$ tinha esse objetivo, porque, ao manter a umidade relativa do ar no valor nominal de $76 \%$, esperava-se redução na velocidade e maior uniformidade no processo de absorção de água pelas sementes. Esse efeito tem sido observado em outras espécies, entre as quais o pimentão (Panobianco e Marcos Filho, 1998), a cenoura (Rodo et al., 2000) e a soja (Marcos Filho et al., 2001). Essa menor absorção de água pode reduzir o desenvolvimento de fungos nas sementes durante a execução do teste, além de promover o envelhecimento mais lento das sementes, pois, o maior efeito passa a ser da temperatura e do período de exposição sem, contudo, provocar a aceleração da deterioração em função da elevação do teor de água a níveis críticos para as sementes.

Por outro lado, quando se utilizou água, a umidade das sementes ao final do período de envelhecimento foi maior, atingindo valores médios de 22,6 a 26,1\% (Tabela 4), apresentando também maior variabilidade entre lotes. Valores finais de umidade elevados e menos uniformes se constituem nas principais dificuldades desse teste, motivo gerador da proposta alternativa de deterioração controlada, na qual se pode fixar o teor de água desejado nas sementes, reduzindo-se também sua variabilidade entre os lotes em comparação. Isso indica que, quanto menor a variação no teor de água, menor o prejuízo à germinação, inferindo 
assim, menor taxa de deterioração sob essa condição (Rodo et al., 2000; Panobianco e Marcos Filho, 2001).

O comportamento dos lotes no teste de envelhecimento acelerado com água e solução saturada de $\mathrm{NaCl}$ (Tabela 5) variou em função da substância umidificadora das minicâmaras e também das condições de tempo-temperatura de exposição. Pelo método tradicional (com água), houve reduções drásticas na germinação das sementes, as quais perderam totalmente a vitalidade quando expostas a $45^{\circ} \mathrm{C}$ por 72h. Mesmo nas condições menos severas de envelhecimento, $41^{\circ} \mathrm{C} / 48 \mathrm{~h}$, a maior parte dos lotes teve a germinação diminuída em cerca de 50 pontos percentuais em relação aos valores iniciais. Não obstante a drasticidade dos efeitos, o envelhecimento a $41^{\circ} \mathrm{C} / 48 \mathrm{~h}$ e a $42^{\circ} \mathrm{C} / 60 \mathrm{~h}$ permitiu classificar os lotes em quatro e três níveis de vigor, respectivamente, no entanto, sem consistência entre eles no ordenamento dos lotes. No caso da condição $41^{\circ} \mathrm{C} / 48 \mathrm{~h}$, houve concordância parcial com a maioria dos resultados do teste de deterioração controlada, ao enquadrar o lote 8 na classe de menor vigor e o lote 1 na classe de maior vigor.

TABELA 5. Porcentagem de germinação de oito lotes de sementes após envelhecimento acelerado (EA) com $\mathrm{H}_{2} \mathrm{O}$ e solução saturada de $\mathrm{NaCl}$, em três combinações de temperatura e períodos de exposição.

\begin{tabular}{|c|c|c|c|c|c|c|}
\hline & \multicolumn{3}{|c|}{$\mathrm{EA}-\mathrm{H}_{2} \mathrm{O}$} & \multicolumn{3}{|c|}{$\mathrm{EA}-\mathrm{NaCl}$} \\
\hline & $41^{\circ} \mathrm{C} / 48 \mathrm{~h}$ & $42^{\circ} \mathrm{C} / 60 \mathrm{~h}$ & $45^{\circ} \mathrm{C} / 72 \mathrm{~h}$ & $41^{\circ} \mathrm{C} / 48 \mathrm{~h}$ & $42^{\circ} \mathrm{C} / 60 \mathrm{~h}$ & $45^{\circ} \mathrm{C} / 72 \mathrm{~h}$ \\
\hline 1 & $62 \mathrm{~A}$ & $34 \mathrm{C}$ & 0,0 & $76 \mathrm{~A}$ & $63 C$ & $65 \mathrm{~B}$ \\
\hline 2 & $53 \mathrm{~B}$ & $37 \mathrm{~B}$ & 0,0 & $76 \mathrm{~A}$ & $65 C$ & $61 B$ \\
\hline 3 & $40 \mathrm{C}$ & $28 \mathrm{C}$ & 0,0 & $75 \mathrm{~A}$ & $69 B$ & $67 \mathrm{~A}$ \\
\hline 4 & $43 \mathrm{C}$ & $33 \mathrm{C}$ & 0,0 & $76 \mathrm{~A}$ & $69 B$ & $70 \mathrm{~A}$ \\
\hline 5 & $42 \mathrm{C}$ & $40 B$ & 0,0 & 77A & 77A & $62 \mathrm{~B}$ \\
\hline 6 & $41 \mathrm{C}$ & $51 \mathrm{~A}$ & 0,0 & 74A & $52 \mathrm{D}$ & $63 \mathrm{~B}$ \\
\hline 7 & $37 \mathrm{C}$ & $32 \mathrm{C}$ & 0,0 & $67 \mathrm{~B}$ & $69 B$ & $72 \mathrm{~A}$ \\
\hline 8 & 31D & $34 \mathrm{C}$ & 0,0 & $69 \mathrm{~B}$ & $63 C$ & $59 \mathrm{~B}$ \\
\hline
\end{tabular}

Em cada coluna, médias seguidas pela mesma letra não diferem entre si pelo teste de Scott e Knott em 10\% de probabilidade.

Quando se utilizou solução saturada de $\mathrm{NaCl}$ (Tabela 5), o envelhecimento das sementes, medido pela germinação, foi menos drástico que no procedimento tradicional com água, sendo proporcional ao aumento da temperatura e tempo de exposição. Mesmo na condição mais severa, que foi a exposição a $45^{\circ} \mathrm{C} / 72 \mathrm{~h}$, a germinação ficou, na média dos lotes, semelhante à obtida nas condições intermediárias do teste de deterioração controlada e superior à dos testes de germinação a baixa temperatura e emergência a campo. $\mathrm{O}$ número de classes formadas e o ordenamento dos lotes variaram de acordo com a temperatura-tempo de exposição. A $42^{\circ} \mathrm{C} / 60 \mathrm{~h}$ houve maior estratificação dos lotes, no entanto, houve divergência dos resultados anteriores ao não posicionar o lote 8 dentro do grupo de pior vigor e ao enquadrar o lote 5 , isoladamente, como o de maior vigor. Além disso, o tempo de exposição de $60 \mathrm{~h}$ apresenta inconveniente de ordem prática para sua aplicação, exigindo que o analista execute o teste fora do horário de trabalho. O envelhecimento a $41^{\circ} \mathrm{C} / 48 \mathrm{~h}$, embora pouco drástico, foi suficiente para designar os lotes 7 e 8 como de menor vigor, podendo se constituir numa alternativa de estudos visando a classificação para lotes de vigor intermediário.

A heterogeneidade no ordenamento dos lotes, pelos testes adotados, remete às considerações de que o vigor é derivado da interação entre vários atributos, presentes nas sementes em graus diferentes, com respostas variadas em função do tipo e intensidade do estresse imposto. Assim é que se torna mais fácil e confiável a separação de lotes de sementes com o alto e o baixo vigor. Por outro lado, a classificação dos lotes de vigor intermediários, como na presente pesquisa, fica muito dependente das condições do teste. Para esse caso, os resultados obtidos permitem indicar que condições menos drásticas de envelhecimento possam se constituir em alternativas, como o uso da deterioração controlada com teor de água nas sementes ajustado entre 15 e $21 \%$, temperatura entre 41 e $43^{\circ} \mathrm{C}$ e período de exposição de $24 \mathrm{~h}$. De forma semelhante, o uso de solução saturada de $\mathrm{NaCl}$ nas minicâmaras, no teste de envelhecimento acelerado, pode permitir separação útil entre lotes de vigor intermediário.

Cabe ressaltar que, pelo teste de emergência a campo, normalmente utilizado como referência para os resultados dos testes de vigor, foi verificado que os lotes de qualidade 
inferior foram os lotes 7 e 8, da mesma forma como no teste de germinação a baixa temperatura. Também, os lotes 2 e 3 apresentaram menores potenciais quando analisados pelos testes de germinação a baixa temperatura e de emergência em campo, respectivamente. De acordo com a AOSA (1983), dentre outras características, o teste de vigor deve apresentar relação com a emergência de plântulas em campo. Portanto, podem ser considerados como adequados para avaliação do vigor de sementes de algodoeiro, a combinação $15 \%$ de umidade das sementes e temperatura de $43^{\circ} \mathrm{C}$, no teste de deterioração controlada e $41^{\circ} \mathrm{C} / 48 \mathrm{~h}$ no teste de envelhecimento acelerado com solução salina de $\mathrm{NaCl}$.

\section{CONCLUSÕES}

Os testes mais promissores para a avaliação do potencial fisiológico de sementes de algodoeiro são a deterioração controlada com o teor de água das sementes ajustado para $15 \%$ a $43^{\circ} \mathrm{C}$ por $24 \mathrm{~h}$ e o envelhecimento acelerado com solução saturada de $\mathrm{NaCl}$, conduzido a $41^{\circ} \mathrm{C} / 48 \mathrm{~h}$.

$\mathrm{O}$ envelhecimento acelerado tradicional a $45^{\circ} \mathrm{C} / 72 \mathrm{~h}$ mostrou-se muito drástico, provocando a perda da viabilidade das sementes.

\section{REFERÊNCIAS}

ASSOCIATION OS OFFICIAL SEED ANALYSTIS AOSA. Seed vigour testing handbook. East Lansing: AOSA, 1983. 93p. (Contribuition, 32).

BARROS, A.S.R.; DIAS, M.C.L. Aferição de testes de vigor para sementes de milho. Informativo ABRATES, Londrina, v.2, n.4, p.10-22, 1992.

BENNETT, M.A.; BARR, A.J.; GRASSBAUGH, E.M.; EVANS, A.F. Seed vigor evaluation of $s u$, se and $s h 2$ sweet corn genotypes using the saturated salt accelerated aging (SSAA) test. In: CONGRESS OF ISTA, 25. Pretoria, Abstracts... Pretoria, 1998. p.92-93.

BRASIL. Ministério da Agricultura e Reforma Agrária. Regras para análise de sementes. Brasília: SNDA/DNDV/ CLAV, 1992. 365p.

CESM. Comissão Estadual de Sementes e Mudas - Normas e padrões de sementes do Estado de Mato Grosso, MAPA/ DFA. Cuiabá, 2005. 90p.

DIAS, M.C.L.; BARROS, A.S.R. Aferição de testes de vigor para sementes de feijão. Informativo ABRATES, Londrina, v.3, n.1, p.7-23, 1992.

HAMPTON, J.G.; COOLBEAR, P. Potential versus actual seed performance can vigour testing provide an answer? Seed Science and Technology, Zurich, v.18, n.2, p.215228, 1990.

HAMPTON, J.G.; TEKRONY, D.M. Handbook of vigor test methods. Zurich: ISTA, 1995a, 117p.

HAMPTON, J. G.; TEKRONY, D.M. Controlled deterioration test. In: HAMPTON, J.G.; TEKRONY, D.M. Handbook of vigour test methods. Zurich: ISTA, $1995 \mathrm{~b}$. p.70-78.

INTERNATIONAL SEED TESTING ASSOCIATION ISTA. Handbook of vigour test methods. Zurich: ISTA, 1995. p. $35-50$

IRIGON, D.L.; ROSSINI, M.C. Aferição de testes de vigor para sementes de trigo. Informativo ABRATES, Londrina, v.2, n.2, p.7-16, 1992.

JIANHUA, Z.; McDONALD, M.B. The saturated salt accelerated aging test for small-seeded crops. Seed Science and Technology, Zurich, v.25, p.123-131, 1996.

KRZYZANOWSKI, F.C.; FRANÇA NETO, J.B. Situação atual do uso de testes de vigor como rotina em programas de sementes no Brasil. Informativo ABRATES, Londrina, v.1, n.3, p.42-53, 1991.

KRZYZANOWSKI, F.C.; VIEIRA, R.D. Deterioração controlada. In: KRZYZANOSWKI, F.C.; VIEIRA, R.D.; FRANÇA NETO. J.B. Vigor de sementes: conceitos e testes. Londrina: ABRATES. 1999. 218p.

LAPOSTA, J.A. Comparação entre métodos para avaliação da qualidade fisiológica de sementes de algodão (Gossypium hirsutum L). Lavras, 1991. 61p. Dissertação (Mestrado em Agronomia - Fitotecnia) - Escola Superior de Agricultura de Lavras, Lavras.

MARCOS FILHO, J. Fisiologia de sementes de plantas cultivadas. Piracicaba: FEALQ. 495p. 2005.

MARCOS FILHO, J. Testes de vigor: importância e utilização. In: KRZYZANOWSKI, F.C.; VIEIRA, R.D.; FRANÇA NETO, J.B. Vigor de sementes: conceitos e testes. Londrina: ABRATES, 1999a. p.1.1-1.2.

MARCOS FILHO, J. Teste de envelhecimento acelerado. In: KRZYZANOWSKI, F.C.; VIEIRA, R.D.; FRANÇA NETO, J.B (Ed.). Vigor de sementes: conceitos e testes. Londrina: ABRATES, 1999b. p.1-24.

MARCOS FILHO, J.; NOVEMBRE, A.D.C.; PESCARIN, H.M.C. Testes de envelhecimento acelerado e de deterioração 
controlada para avaliação do vigor de sementes de soja. Scientia Agricola, Piracicaba, v.58, n.2, p.421-426, 2001.

MENDONÇA, E.A.F.; RAMOS, N.P.; FESSEL, S.A.; SADER, R. Teste de deterioração controlada em sementes de brócolis (Brassica oleraceae L.). var. Itálica. Revista Brasileira de Sementes, Brasília, v.22, n.1, p.280-287, 2000 .

NAKAGAWA, J. Testes de vigor baseados no desempenho de plântulas. In: KRZYZANOWSKI, F.C.; VIEIRA, R.D.; FRANÇA NETO, J.B. Vigor de sementes: conceitos e testes. Londrina: ABRATES, 1999. p.2.1-2.24.

PANOBIANCO, M.; MARCOS FILHO, J. Envelhecimento acelerado e deterioração controlada em sementes de tomate. Scientia Agricola, Piracicaba, v.58, n.3, p.525-531, 2001.

PANOBIANCO, M.; MARCOS FILHO, J. Comparação entre métodos para avaliação da qualidade fisiológica de sementes de pimentão. Revista Brasileira de Sementes, Brasília, v.20, n.2, p.306-310, 1998.

POWELL, A. A. The controlled deterioration test. In: VAN DER VENTER, H.A. (Ed.) Seed vigour testing seminar. Copenhagen: ISTA, 1995. p.73-87.

POWELL, A.A.; MATTHEWS, S. Evaluation of controlled deterioration: a new vigour test for small seed vegetable. Seed Science and Technology, Zurich, v.9, n.22, p.663-640, 1981.

RODO, A.B.; PANOBIANCO, M.; MARCOS FILHO, J. Metodologia alternativa do teste de envelhecimento acelerado para sementes de cenoura. Scientia Agricola, Piracicaba, v.57, n.2, p.289-292, 2000.

ROSSETO, C.A.V.; MARCOS FILHO, J. Comparação entre métodos de envelhecimento acelerado e deterioração controlada para avaliação da qualidade fisiológica de sementes de soja. Scientia Agrícola, Piracicaba, v.52, n.1, p. 123-131, 1995.

ROSSETO, C.A.V.; LIMA, T.M.; GUIMARÃES, E.C. envelhecimento acelerado e deterioração controlada em sementes de amendoim. Pesquisa Agropecuária Brasileira, Brasília, v.39, p.795-801, 2004.

SANTOS, C.M.R.; MENEZES, N.L.; VILLELA, F.A. Teste de deterioração controlada para a avaliação do vigor de sementes de feijão. Revista Brasileira de Sementes, Pelotas, v.25, p.28-35, 2003.

TEKRONY, D.M. Accelerated aging test. In: HAMPTON, J.G.; TEKRONY, D.M. (Ed.). Handbook of vigour test methods. 3.ed. Zurich: International Seed Testing Association, 1995. p.35-50.

TORRES, S.B. Comparação entre testes de vigor para avaliar a qualidade fisiológica de sementes de algodão. Revista Brasileira de Sementes, Brasília, v.20, n.2, p.249-253, 1998.

TORRES, S.B. Métodos para a avaliação do potencial fisiológico de sementes de melão. Piracicaba. 2002. 103f. Tese (Doutorado em Agronomia), Escola Superior de Agricultura "Luiz de Queiroz", Universidade de São Paulo, 2002. 